\title{
HOMOGÉNÉISATION D'UN PROBLÈME OSCILLANT DE CONDUCTION THERMIQUE
}

\author{
A. Ait Moussa ${ }^{1}$ And J. Messaho ${ }^{1}$
}

\begin{abstract}
We study the asymptotic behavior of a thermal problem, of a containing structure, an oscillating thin layer of thickness and conductivity depending of $\varepsilon$. We use the the epi-convergence method to find the limit problems with interface conditions. The obtained results are tested numerically.

Résumé. On étudie le comportement asymptotique par la méthode d'épiconvergence d'un problème thermique, d'une structure contenant une bande oscillante d'épaisseur et de conductivité dépendant de $\varepsilon$. On trouve les problèmes limites avec conditions d'interfaces. Les résultats obtenus sont testés numériquement.
\end{abstract}

\section{INTRODUCTION}

En physique mathématique, on rencontre plusieurs sortes de problèmes aux limites, conduction de la chaleur, électrostatique, électromagnétique, mécanique des milieux continus, où l'inconnu $u$ satisfait à la surface de séparation entre deux domaines $\Omega_{1}$ et $\Omega_{2}$ à des conditions de transmission de type:

$$
\begin{aligned}
u_{\left.\right|_{\Omega_{1}}} & =u_{\left.\right|_{\Omega_{2}}} \\
\left.\sigma_{1}|\nabla u|^{p-2} \frac{\partial u}{\partial n}\right|_{\Omega_{1}} & =\left.\sigma_{2}|\nabla u|^{q-2} \frac{\partial u}{\partial n}\right|_{\Omega_{2}}
\end{aligned}
$$

où $p>1, q>1$ et $n$ représente la normale extérieur à la surface de séparation, $\sigma_{1}$ et $\sigma_{2}$ des constantes associées à chacun des domaines $\Omega_{1}$ et $\Omega_{2}$, les conditions aux limites de type (1) et (2) sont rencontrées dans des problèmes de conductivité thermique où $\sigma_{1}$ et $\sigma_{2}$ désignent les conductivités de deux corps, ou dans des problèmes d'électrostatique ou magnétostatique où $\sigma_{1}$ et $\sigma_{2}$ sont les constantes diélectriques ou les perméabilités, un problème avec conditions de transmission de type (1) et (2), le cas $p=2$ et $q=2$, est traité par E. SANCHEZPALENCIA dans [7].

L'objet de ce travail est d'étudier le comportement asymptotique de solutions d'un problème de conductivité thermique. Ce dernier est posé dans une structure contenant une bande oscillante d'épaisseur et de conductivité dépendant aussi de $\varepsilon, \varepsilon$ étant un paramètre destiné à tendre vers 0 .

Des problèmes similaires sont dans Brillard et al [4]. Le cas vectoriel on le trouve dans A. Ait Moussa et al et Brillard et al dans $[1,5]$. Ce papier est organisé comme suit:

On énonce le problème à étudier dans la section 1, et on définit des espaces fonctionnels pour cette étude dans la section 2. Dans la section 3 , on étudie le problème $\left(\mathcal{P}_{\varepsilon}\right)$. La section 4 est réservée à la détermination

\footnotetext{
1 Département de Mathématiques et Informatique, Faculté des Sciences, Université Mohammed 1er, Oujda;

e-mail: moussa@sciences.univ-oujda.ac.ma, \& j_messaho@yahoo.fr
}

(C) EDP Sciences, SMAI 2007 
du problème limite. Enfin dans la section 5 on donnera un test numérique illustrant les résultats théoriques obtenus.

\section{Position du PRoBlÈme}

On considère un problème de conduction thermique non linéaire dans un corps occupant un domaine $\Omega \subset \mathbb{R}^{3}$, de frontière Lipschitzienne $\partial \Omega$, composé d'une bande $B_{\varepsilon}$, à frontière oscillante $\Sigma_{\varepsilon}^{ \pm}$, d'interface moyenne $\Sigma$, de conductivité très élevée, et d'une région $\Omega_{\varepsilon}$ avec une conductivité constante (voir figure 1.1). La structure, occupant le domaine $\Omega$, est soumise à une température extérieure $f . f: \Omega \longrightarrow \mathbb{R}$, et refroidie au bord $\partial \Omega$.

Les équations du problème sont :

$$
\left(\mathcal{P}^{\varepsilon}\right)\left\{\begin{aligned}
\operatorname{div}\left(\left|\nabla u^{\varepsilon}\right|^{p-2} \nabla u^{\varepsilon}\right)+f & =0 & & \operatorname{dans} \Omega_{\varepsilon} \\
\frac{1}{\varepsilon^{\alpha}} \operatorname{div}\left(\left|\nabla u^{\varepsilon}\right|^{q-2} \nabla u^{\varepsilon}\right)+f & =0 & & \operatorname{dans} B_{\varepsilon} \\
{\left[u^{\varepsilon}\right] } & =0 & & \text { sur } \Sigma_{\varepsilon}^{ \pm} \\
\left.\left|\nabla u^{\varepsilon}\right|^{p-2} \frac{\partial u^{\varepsilon}}{\partial n}\right|_{\Omega_{\varepsilon}} & =\left.\frac{1}{\varepsilon^{\alpha}}\left|\nabla u^{\varepsilon}\right|^{q-2} \frac{\partial u^{\varepsilon}}{\partial n}\right|_{B_{\varepsilon}} & & \text { sur } \Sigma_{\varepsilon}^{ \pm} \\
u^{\varepsilon} & =0 & & \text { sur } \partial \Omega
\end{aligned}\right.
$$

Où :

- $n$ la normale extérieure à $\partial \Omega$.

- $p>1, q>1, \alpha \geq 0$.

- $1<r \leq \min (p, q)$ et $r^{\prime}$ son conjugué.

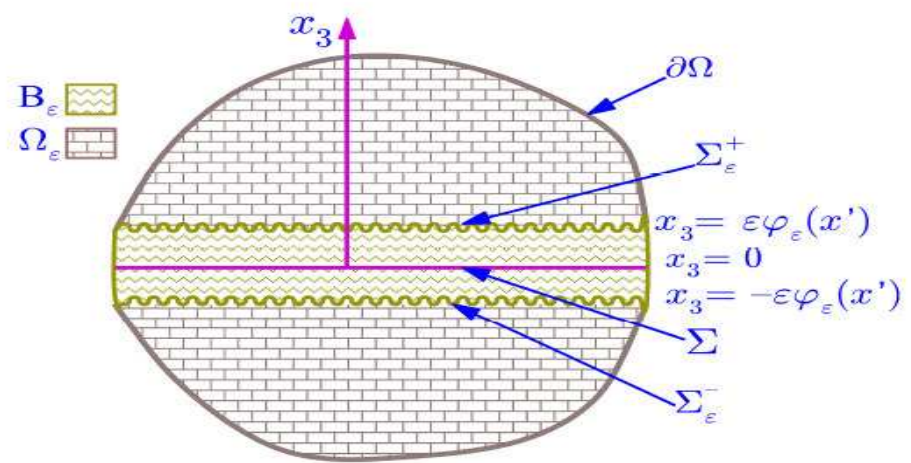

Figure 1.1. Domaine $\Omega$

Où $\varepsilon$ étant un paramètre positif destiné à tendre vers zéro, $\varphi_{\varepsilon}$ est une fonction réelle bornée, $] 0, \varepsilon\left[{ }^{2}\right.$-périodique.

\section{Notations ET CADRE FOnCTIONNEL}

\subsection{Notations}

On donne quelques notations qui sera utile dans la suite:

$$
\left\{\begin{array}{l}
x=\left(x^{\prime}, x_{3}\right) \text { où } x^{\prime}=\left(x_{1}, x_{2}\right), \lambda=1,2, \\
\left.\nabla^{\prime}=\left(\frac{\partial}{\partial x_{1}}, \frac{\partial}{\partial x_{2}}\right), Y=\right] 0,1[\times] 0,1[ \\
\varphi: \mathbb{R}^{2} \longrightarrow\left[a_{1}, a_{2}\right] \text { où: }(\varphi \text { est } Y-\text { périodique }) \text { et } a_{2} \geq a_{1}>0 \\
\varphi_{\varepsilon}\left(x^{\prime}\right)=\varphi\left(\frac{x^{\prime}}{\varepsilon}\right), \frac{\partial \varphi}{\partial x_{\lambda}} \in \mathcal{C}(\Sigma) \cap L^{\infty}(\Sigma), \\
m(\varphi)=\left(\frac{1}{\int_{Y} d x^{\prime}}\right) \int_{Y} \varphi\left(x^{\prime}\right) d x^{\prime} \\
\eta(t)=\lim _{\varepsilon \rightarrow 0} \varepsilon^{1-t} \text { avec } t>0 .
\end{array}\right.
$$


- On désignera par $C$ diverses constantes indépendantes de $\varepsilon$.

- On utilise la convention $0 .+\infty=0$.

\subsection{Cadre fonctionnel}

Dans la suite, on introduit l'espace suivant :

$$
V^{\varepsilon}=\left\{u \in W_{0}^{1, r}(\Omega) \mid u_{\left.\right|_{\Omega_{\varepsilon}}} \in W^{1, p}\left(\Omega_{\varepsilon}\right), u_{\left.\right|_{B_{\varepsilon}}} \in W^{1, q}\left(B_{\varepsilon}\right)\right\},
$$

c'est un espace de Banach muni de la norme :

$$
u \longmapsto\|u\|_{W^{1, p}\left(\Omega_{\varepsilon}\right)}+\|u\|_{W^{1, q}\left(B_{\varepsilon}\right)} .
$$

Posons :

$$
\begin{aligned}
V^{q}(\Sigma) & =\left\{u \in W_{0}^{1, p} \mid u_{\left.\right|_{\Sigma}} \in W^{1, q}(\Sigma)\right\} \\
V^{C}(\Sigma) & =\left\{u \in W_{0}^{1, p} \mid u_{\left.\right|_{\Sigma}}=C\right\} .
\end{aligned}
$$

$V^{C}(\Sigma)$ est un espace de Banach, muni de la norme de $W_{0}^{1, p}(\Omega)$.

$V^{q}(\Sigma)$ est espace de Banach, muni de la norme suivante :

Posons :

$$
u \longmapsto\|\nabla u\|_{L^{p}(\Omega)^{3}}+\left\|\nabla^{\prime} u_{\left.\right|_{\Sigma}}\right\|_{L^{q}(\Sigma)^{2}}
$$

$$
\begin{aligned}
\mathbb{G}^{\alpha} & =\left\{\begin{array}{lll}
\left\{u \in W_{0}^{1, p}(\Sigma) \mid \eta(\alpha) \nabla^{\prime} u_{\left.\right|_{\Sigma}} \in L^{q}(\Sigma)^{2}\right\} & \text { si } & \alpha \leq 1 \\
V^{C} & \text { si } & \alpha>1 .
\end{array}\right. \\
\mathbb{D}^{\alpha} & =\left\{\begin{array}{lll}
\mathcal{D}(\Omega) & \text { si } & \alpha \leq 1 \\
\left\{u \in \mathcal{D}(\Omega) \mid u_{\left.\right|_{\Sigma}}=C\right\} & \text { si } & \alpha>1
\end{array}\right.
\end{aligned}
$$

Notre but, dans ce travail, est d'étudier le problème $\left(\mathcal{P}^{\varepsilon}\right)$, ainsi que son comportement asymptotique.

\section{Etude DU PRoblème $\left(\mathcal{P}^{\varepsilon}\right)$}

Le problème $\left(\mathcal{P}^{\varepsilon}\right)$ est équivalent au problème de minimisation suivant :

$$
\left(\Pi^{\varepsilon}\right) \inf _{v \in V^{\varepsilon}}\left\{\frac{1}{p} \int_{\Omega_{\varepsilon}}|\nabla v|^{p}+\frac{1}{q \varepsilon^{\alpha}} \int_{B_{\varepsilon}}|\nabla v|^{q}-\int_{\Omega} f . v\right\} .
$$

Proposition 3.1. Pour $f \in L^{r^{\prime}}(\Omega)$, le problème $\left(\Pi^{\varepsilon}\right)$ admet une unique solution $u^{\varepsilon}$ dans $V^{\varepsilon}$.

Proof. L'existence et l'unicité du minimum $u^{\varepsilon}$ du problème $\left(\Pi^{\varepsilon}\right)$, découle d'un résultat classique (cf., [3, Théorème V.15, p. 88]).

Lemme 3.1. Pour tout $f \in L^{r^{\prime}}(\Omega), p>1$ et $q>1$, la famille $\left(u^{\varepsilon}\right)_{\varepsilon>0}$ satisfait $\grave{a}$ :

$$
\begin{aligned}
\left\|\nabla u^{\varepsilon}\right\|_{L^{p}\left(\Omega_{\varepsilon}\right)}^{p} & \leq C, \\
\frac{1}{\varepsilon^{\alpha}}\left\|\nabla u^{\varepsilon}\right\|_{L^{q}\left(B_{\varepsilon}\right)}^{q} & \leq C .
\end{aligned}
$$

De plus $u^{\varepsilon}$ est bornée dans $W_{0}^{1, r}(\Omega)$. 
Proof. Comme $u^{\varepsilon}$ est la solution du problème $\left(\Pi^{\varepsilon}\right)$, alors on a :

$$
\int_{\Omega_{\varepsilon}}\left|\nabla u^{\varepsilon}\right|^{p-2} \nabla u^{\varepsilon} \nabla v+\frac{1}{\varepsilon^{\alpha}} \int_{B_{\varepsilon}}\left|\nabla u^{\varepsilon}\right|^{q-2} \nabla u^{\varepsilon} \nabla v=\int_{\Omega} f v, \quad \forall v \in V^{\varepsilon} .
$$

En particulier pour $v=u^{\varepsilon}$, on obtient :

$$
\left\|\nabla u^{\varepsilon}\right\|_{L^{p}\left(\Omega_{\varepsilon}\right)}^{p}+\frac{1}{\varepsilon^{\alpha}}\left\|\nabla u^{\varepsilon}\right\|_{L^{q(B \varepsilon)}}^{q}=\int_{\Omega} f u^{\varepsilon}
$$

En vertu des inégalités de Hölder et de Young, on a :

$$
\begin{aligned}
\left\|\nabla u^{\varepsilon}\right\|_{L^{p}\left(\Omega_{\varepsilon}\right)}^{p}+\frac{1}{\varepsilon^{\alpha}}\left\|\nabla u^{\varepsilon}\right\|_{L^{q}\left(B_{\varepsilon}\right)}^{q} & \leq C\left\|\nabla u^{\varepsilon}\right\|_{L^{r}(\Omega)} \\
& \leq C\left(\left\|\nabla u^{\varepsilon}\right\|_{L^{r}\left(\Omega_{\varepsilon}\right)}+\left\|\nabla u^{\varepsilon}\right\|_{L^{r}\left(B_{\varepsilon}\right)}\right) \\
& \leq C\left(\left\|\nabla u^{\varepsilon}\right\|_{L^{p}\left(\Omega_{\varepsilon}\right)}+\left\|\nabla u^{\varepsilon}\right\|_{L^{q}\left(B_{\varepsilon}\right)}\right) \\
& \leq C+\frac{1}{p}\left\|\nabla u^{\varepsilon}\right\|_{L^{p}\left(\Omega_{\varepsilon}\right)}^{p}+\frac{1}{q}\left\|\nabla u^{\varepsilon}\right\|_{L^{q}\left(B_{\varepsilon}\right)}^{q} \\
& \leq C+\frac{1}{p}\left\|\nabla u^{\varepsilon}\right\|_{L^{p}\left(\Omega_{\varepsilon}\right)}^{p}+\frac{1}{\varepsilon^{\alpha}} \frac{1}{q}\left\|\nabla u^{\varepsilon}\right\|_{L^{q}\left(B_{\varepsilon}\right)}^{q}
\end{aligned}
$$

Comme $\frac{1}{p}<1$ et $\frac{1}{q}<1$, donc :

$$
\left\|\nabla u^{\varepsilon}\right\|_{L^{p}\left(\Omega_{\varepsilon}\right)}^{p}+\frac{1}{\varepsilon^{\alpha}}\left\|\nabla u^{\varepsilon}\right\|_{L^{q}\left(B_{\varepsilon}\right)}^{q} \leq C
$$

On aura donc les assertions (3.1), (3.2).

Comme $r \leq \min (p, q)$ et en vertu de (3.1), (3.2), la solution $\left(u^{\varepsilon}\right)$ est bornée dans $W_{0}^{1, r}(\Omega)$.

Définissons l'opérateur " $m{ }^{\varepsilon "}$ qui transforme les fonctions définies sur $B_{\varepsilon}$ en fonctions définies sur $\Sigma$ par :

$$
m^{\varepsilon} u\left(x_{1}, x_{2}\right)=\frac{1}{2 \varepsilon \varphi_{\varepsilon}} \int_{-\varepsilon \varphi_{\varepsilon}}^{\varepsilon \varphi_{\varepsilon}} u\left(x_{1}, x_{2}, x_{3}\right) d x_{3}
$$

Lemme 3.2. L'opérateur " $m^{\varepsilon "}$ défini par (3.3) est linéaire et borné de $L^{q}\left(B_{\varepsilon}\right)$ (resp. $\left.W^{1, q}\left(B_{\varepsilon}\right)\right)$ dans $L^{q}(\Sigma)$ (resp. $\left.W^{1, q}(\Sigma)\right)$, avec norme $\leq C \varepsilon^{-\frac{1}{q}}$, de plus, pour tout $u \in W^{1, q}\left(B_{\varepsilon}\right)$, on $a$ :

$$
\| m^{\varepsilon} u-u_{\left.\right|_{\Sigma} \|_{\left.\right|_{L^{q}(\Sigma)}}^{q}} \leq C \varepsilon^{q-1} \int_{B_{\varepsilon}}|\nabla u|^{q}
$$

Proof. On a:

$$
\int_{\Sigma}\left|m^{\varepsilon} u\right|^{q} d x_{1} d x_{2}=\int_{\Sigma}\left(\frac{1}{2 \varepsilon \varphi_{\varepsilon}}\right)^{q}\left|\int_{-\varepsilon \varphi_{\varepsilon}}^{\varepsilon \varphi_{\varepsilon}} u d x_{3}\right|^{q} d x_{1} d x_{2}
$$

Comme $0<a_{1} \leq \varphi_{\varepsilon} \leq a_{2}$, et en vertu de l'inégalité de Hölder, (3.5) devient :

$$
\begin{aligned}
\int_{\Sigma}\left|m^{\varepsilon} u\right|^{q} d x_{1} d x_{2} & \leq \int_{\Sigma} \frac{1}{2 \varepsilon \varphi_{\varepsilon}}\left(\int_{-\varepsilon \varphi_{\varepsilon}}^{\varepsilon \varphi_{\varepsilon}}|u|^{q} d x_{3}\right) d x_{1} d x_{2} \\
& \leq \frac{1}{2 \varepsilon a_{1}} \int_{\Sigma}\left(\int_{-\varepsilon \varphi_{\varepsilon}}^{\varepsilon \varphi_{\varepsilon}}|u|^{q} d x_{3}\right) d x_{1} d x_{2}
\end{aligned}
$$


Comme $u \in L^{q}\left(B_{\varepsilon}\right)$, et d'après (3.6), on a $m^{\varepsilon} u \in L^{q}(\Sigma)$.

Soit $u \in \mathcal{D}\left(\overline{B_{\varepsilon}}\right)$ on a :

$$
\begin{aligned}
\frac{\partial}{\partial x_{\lambda}}\left(m^{\varepsilon} u\right)\left(x_{1}, x_{2}\right) & =\frac{1}{2} \frac{\partial}{\partial x_{\lambda}}\left(\int_{-1}^{1} u\left(x_{1}, x_{2}, x_{3} \varepsilon \varphi_{\varepsilon}\right) d x_{3}\right) \\
& =\frac{1}{2}\left(\int_{-1}^{1} \frac{\partial u}{\partial x_{\lambda}}\left(x_{1}, x_{2}, x_{3} \varepsilon \varphi_{\varepsilon}\right)+\varepsilon x_{3} \frac{\partial \varphi_{\varepsilon}}{\partial x_{\lambda}} \frac{\partial u}{\partial x_{3}}\left(x_{1}, x_{2}, x_{3} \varepsilon \varphi_{\varepsilon}\right) d x_{3}\right) \\
& =\frac{1}{2 \varepsilon \varphi_{\varepsilon}}\left(\int_{-\varepsilon \varphi_{\varepsilon}}^{\varepsilon \varphi_{\varepsilon}} \frac{\partial u}{\partial x_{\lambda}}+\left(\frac{x_{3}}{\varepsilon \varphi_{\varepsilon}}\right)\left(\varepsilon \frac{\partial \varphi_{\varepsilon}}{\partial x_{\lambda}}\right) \frac{\partial u}{\partial x_{3}} d x_{3}\right) .
\end{aligned}
$$

Donc :

$$
\begin{aligned}
\int_{\Sigma}\left|\frac{\partial}{\partial x_{\lambda}}\left(m^{\varepsilon} u\right)\right|^{q} & =\int_{\Sigma}\left|\frac{1}{2 \varepsilon \varphi_{\varepsilon}}\left(\int_{-\varepsilon \varphi_{\varepsilon}}^{\varepsilon \varphi_{\varepsilon}} \frac{\partial u}{\partial x_{\lambda}}+\left(\frac{x_{3}}{\varepsilon \varphi_{\varepsilon}}\right)\left(\varepsilon \frac{\partial \varphi_{\varepsilon}}{\partial x_{\lambda}}\right) \frac{\partial u}{\partial x_{3}} d x_{3}\right)\right|^{q} \\
& \leq \frac{1}{2 \varepsilon a_{1}} \int_{\Sigma}\left(\int_{-\varepsilon \varphi_{\varepsilon}}^{\varepsilon \varphi_{\varepsilon}}\left|\frac{\partial u}{\partial x_{\lambda}}+\left(\frac{x_{3}}{\varepsilon \varphi_{\varepsilon}}\right)\left(\varepsilon \frac{\partial \varphi_{\varepsilon}}{\partial x_{\lambda}}\right) \frac{\partial u}{\partial x_{3}}\right|^{q} d x_{3}\right)
\end{aligned}
$$

Comme $\frac{\partial \varphi}{\partial x_{\lambda}} \in \mathcal{C}(\Sigma) \cap L^{\infty}(\Sigma)$, alors $\varepsilon \frac{\partial \varphi_{\varepsilon}}{\partial x_{\lambda}}$ est bornée, et par conséquent :

$$
\begin{aligned}
\int_{\Sigma}\left|\frac{\partial}{\partial x_{\lambda}}\left(m^{\varepsilon} u\right)\right|^{q} & \leq \frac{C}{\varepsilon} \int_{B_{\varepsilon}}\left(\left|\frac{\partial u}{\partial x_{\lambda}}\right|^{q}+\left|\frac{\partial u}{\partial x_{3}}\right|^{q}\right) d x_{3} \\
& \leq \frac{C}{\varepsilon} \int_{B_{\varepsilon}}|\nabla u|^{q}
\end{aligned}
$$

Par densité de $\mathcal{D}\left(\bar{B}_{\varepsilon}\right)$ dans $W^{1, q}\left(B_{\varepsilon}\right)$, on a pour tout $u \in W^{1, q}\left(B_{\varepsilon}\right)$ :

$$
\int_{\Sigma}\left|\frac{\partial}{\partial x_{\lambda}}\left(m^{\varepsilon} u\right)\right|^{q} \leq \frac{C}{\varepsilon} \int_{B_{\varepsilon}}|\nabla u|^{q}
$$

Soit $u \in \mathcal{D}\left(\overline{B_{\varepsilon}}\right)$, on a :

$$
\left\|m^{\varepsilon} u-u_{\left.\right|_{\Sigma}}\right\|_{L^{q}(\Sigma)}^{q}=\int_{\Sigma}\left|\left(\frac{1}{2 \varepsilon \varphi_{\varepsilon}} \int_{-\varepsilon \varphi_{\varepsilon}}^{\varepsilon \varphi_{\varepsilon}} u\left(x_{1}, x_{2}, x_{3}\right) d x_{3}\right)-u\left(x_{1}, x_{2}, 0\right)\right|^{q} d x_{1} d x_{2},
$$

et d'après l'inégalité de Hölder, (3.7) devient:

$$
\begin{aligned}
\left\|m^{\varepsilon} u-u_{\left.\right|_{\Sigma}}\right\|_{L^{q}(\Sigma)}^{q} & \leq \frac{1}{2 \varepsilon a_{1}} \int_{\Sigma}\left(\int_{-\varepsilon \varphi_{\varepsilon}}^{\varepsilon \varphi_{\varepsilon}}\left|u\left(x_{1}, x_{2}, x_{3}\right)-u\left(x_{1}, x_{2}, 0\right)\right|^{q} d x_{3}\right) d x_{1} d x_{2} \\
& \leq \frac{C}{\varepsilon} \int_{\Sigma}\left(\int_{-\varepsilon \varphi_{\varepsilon}}^{\varepsilon \varphi_{\varepsilon}}\left|\int_{0}^{x_{3}} \frac{\partial u}{\partial x_{3}}\left(x_{1}, x_{2}, t\right) d t\right|^{q} d x_{3}\right) d x_{1} d x_{2} \\
& \leq \frac{C}{\varepsilon} \int_{\Sigma}\left(\int_{-\varepsilon \varphi_{\varepsilon}}^{\varepsilon \varphi_{\varepsilon}}\left|x_{3}\right|^{q-1}\left(\int_{-\varepsilon \varphi_{\varepsilon}}^{\varepsilon \varphi_{\varepsilon}}\left|\frac{\partial u}{\partial x_{3}}\left(x_{1}, x_{2}, t\right)\right|^{q} d t\right) d x_{3}\right) d x_{1} d x_{2} \\
& \leq C \varepsilon^{q-1} \int_{\Sigma}\left(\int_{-\varepsilon \varphi_{\varepsilon}}^{\varepsilon \varphi_{\varepsilon}}\left|\frac{\partial u}{\partial x_{3}}\right|^{q} d x_{3}\right) d x_{1} d x_{2} \\
& \leq C \varepsilon^{q-1} \int_{B_{\varepsilon}}|\nabla u|^{q} d x
\end{aligned}
$$


Par densité de $\mathcal{D}\left(\overline{B_{\varepsilon}}\right)$ dans $W^{1, q}\left(B_{\varepsilon}\right)$, on aura pour tout $u \in W^{1, q}\left(B_{\varepsilon}\right)$ :

$$
\left\|m^{\varepsilon} u-u_{\left.\right|_{\Sigma}}\right\|_{L^{q(\Sigma)}}^{q} \leq C \varepsilon^{q-1} \int_{B_{\varepsilon}}|\nabla u|^{q} d x
$$

D'où la preuve du lemme 3.2.

Lemme 3.3. Toute suite $\left(u^{\varepsilon}\right)_{\varepsilon>0} \subset V^{\varepsilon}$, vérifiant (3.1), (3.2), satisfait à :

$$
\left\|\nabla^{\prime}\left(m^{\varepsilon} u^{\varepsilon}\right)\right\|_{\left(L^{q}(\Sigma)\right)^{2}}^{q} \leq C \varepsilon^{\alpha-1} .
$$

De plus $m^{\varepsilon} u^{\varepsilon}$ est bornée dans $L^{r}(\Sigma)$.

Proof. En vertu du lemme 3.2, on a :

$$
\left\|\frac{\partial\left(m^{\varepsilon} u^{\varepsilon}\right)}{\partial x_{\lambda}}\right\|_{L^{q}(\Sigma)^{2}}^{q} \leq C \varepsilon^{-1} \int_{B_{\varepsilon}}\left|\nabla u^{\varepsilon}\right|^{q} d x
$$

d'après (3.2), on a:

$$
\left\|\frac{\partial\left(m^{\varepsilon} u^{\varepsilon}\right)}{\partial x_{\lambda}}\right\|_{L^{q(\Sigma)^{2}}}^{q} \leq C \varepsilon^{\alpha-1}
$$

d'où (3.8) .

Montrons que $\left(m^{\varepsilon} u^{\varepsilon}\right)$ est bornée dans $L^{r}(\Sigma)$.

D'après (3.4), (cf, lemme 3.2), on a :

$$
\left\|m^{\varepsilon} u^{\varepsilon}-\left.u^{\varepsilon}\right|_{\Sigma}\right\|_{L^{q}(\Sigma)}^{q} \leq C \varepsilon^{q-1} \int_{B_{\varepsilon}}\left|\nabla u^{\varepsilon}\right|^{q} d x
$$

en vertu de (3.2), on obtient :

$$
\left\|m^{\varepsilon} u^{\varepsilon}-u_{\mid \Sigma}^{\varepsilon}\right\|_{L^{q}(\Sigma)}^{q} \leq C \varepsilon^{\alpha+q-1} .
$$

Comme $r \leq q$ et $\Omega$ est borné, alors on a :

$$
\begin{aligned}
\left\|m^{\varepsilon} u^{\varepsilon}-\left.u^{\varepsilon}\right|_{\Sigma}\right\|_{L^{r}(\Sigma)} & \leq C\left\|m^{\varepsilon} u^{\varepsilon}-u_{\mid \Sigma}^{\varepsilon}\right\|_{L^{q}(\Sigma)} \\
& \leq C \varepsilon^{\frac{\alpha+q-1}{q}} .
\end{aligned}
$$

Comme $u^{\varepsilon}$ vérifie (3.1), (3.2), alors on a $u^{\varepsilon}$ est bornée dans $W_{0}^{1, r}(\Omega)$, donc il existe $u^{*} \in W_{0}^{1, r}(\Omega)$ tel que pour une sous suite de $u^{\varepsilon}$, notée encore $u^{\varepsilon}, u^{\varepsilon} \rightarrow u^{*}$ dans $W^{1, r}(\Omega)$, donc $\left.u^{\varepsilon}\right|_{\Sigma}$ est bornée dans $L^{r}(\Sigma)$.

Comme :

$$
\left\|m^{\varepsilon} u^{\varepsilon}\right\|_{L^{r}(\Sigma)} \leq\left\|m^{\varepsilon} u^{\varepsilon}-\left.u^{\varepsilon}\right|_{\Sigma}\right\|_{L^{r}(\Sigma)}+\left\|\left.u^{\varepsilon}\right|_{\Sigma}\right\|_{L^{r}(\Sigma)}
$$

d'après (3.9), (3.10), alors il existe une constante $C>0$ telle que :

$$
\left\|m^{\varepsilon} u^{\varepsilon}\right\|_{L^{r}(\Sigma)}^{r} \leq C
$$

D'où la preuve du lemme 3.3 . 
Proposition 3.2. La solution du problème $\left(\Pi^{\varepsilon}\right),\left(u^{\varepsilon}\right)_{\varepsilon}$, possède une sous suite faiblement convergente vers un élément $u^{*}$ dans $W_{0}^{1, r}(\Omega)$ satisfaisant à :

(1) $u^{*} \in W^{1, p}(\Omega)$.

(2) Si $\alpha=1$ :

$$
u_{\mid \Sigma}^{*} \in W^{1, q}(\Sigma)
$$

(3) Si $\alpha>1$ :

$$
u^{*}{ }_{\Sigma}=C \text {. }
$$

Proof. En vertu du lemme 3.1, on a la suite $u^{\varepsilon}$ est bornée dans $W_{0}^{1, r}(\Omega)$, donc elle possède une sous suite notée encore $u^{\varepsilon}$ qui converge faiblement vers un élément $u^{*}$ dans $W_{0}^{1, r}(\Omega)$, donc

$$
\lim _{\varepsilon \rightarrow 0} \int_{\Omega} \nabla u^{\varepsilon} v=\int_{\Omega} \nabla u^{*} v, \forall v \in L^{r^{\prime}}(\Omega)^{3},
$$

d'après (3.1), on a $\chi_{\Omega_{\varepsilon}} \nabla u^{\varepsilon}$ est bornée dans $L^{p}(\Omega)^{3}$, donc pour une sous suite de $\chi_{\Omega_{\varepsilon}} \nabla u^{\varepsilon}$, notée encore $\chi_{\Omega_{\varepsilon}} \nabla u^{\varepsilon}$, il existe $w \in L^{p}(\Omega)^{3} \subset L^{r}(\Omega)^{3}$, tel que :

$$
\chi_{\Omega_{\varepsilon}} \nabla u^{\varepsilon} \rightarrow w \text { dans } L^{p}(\Omega)^{3},
$$

donc

Soit $v \in L^{r^{\prime}}(\Omega)^{3}$, alors on a :

$$
\chi_{\Omega_{\varepsilon}} \nabla u^{\varepsilon} \rightarrow w \text { dans } L^{r}(\Omega)^{3} .
$$

$$
\int_{\Omega} \nabla u^{\varepsilon} v=\int_{\Omega} \nabla u^{\varepsilon} \chi_{\Omega_{\varepsilon}} v+\int_{\Omega} \nabla u^{\varepsilon} \chi_{B_{\varepsilon}} v
$$

donc par passage à la limite on a :

$$
\int_{\Omega} \nabla u^{*} v=\int_{\Omega} w v+\lim _{\varepsilon \rightarrow 0} \int_{\Omega} \nabla u^{\varepsilon} \chi_{B_{\varepsilon}} v,
$$

or $\chi_{B_{\varepsilon}} v \rightarrow 0$ dans $L^{r^{\prime}}(\Omega)^{3}$, donc

$$
\int_{\Omega} \nabla u^{*} v=\int_{\Omega} w v
$$

donc $\nabla u^{*}=w$, par conséquent $\nabla u^{*} \in L^{p}(\Omega)^{3}$, et en vertu d'un résultat classique (cf, [8]), on a $u^{*} \in W_{0}^{1, p}(\Omega)$. On a :

$$
\left\{\begin{array}{l}
\left\|m^{\varepsilon} u^{\varepsilon}-u_{\left.\right|_{\Sigma}}\right\|_{L^{r}(\Sigma)} \leq C \varepsilon^{\frac{\alpha+q-1}{q}} \\
\left.u_{\left.\right|_{\Sigma}}^{\varepsilon} \rightarrow u^{*}\right|_{\Sigma} \operatorname{dans} L^{r}(\Sigma)
\end{array}\right.
$$

- $\alpha=1$ :

D'après l'estimation (3.8), la suite $\nabla^{\prime} m^{\varepsilon} u^{\varepsilon}$ possède une sous suite notée encore $\nabla^{\prime} m^{\varepsilon} u^{\varepsilon}$ converge faiblement vers un élément $u^{2}$ dans $L^{q}(\Sigma)^{2}$, donc dans $L^{r}(\Sigma)^{2}$, et $m^{\varepsilon} u^{\varepsilon} \rightarrow u^{*}{ }_{\Sigma}$ dans $W^{1, r}(\Sigma)$, alors $\left.\nabla^{\prime} u^{*}\right|_{\Sigma}=u^{2}$, d'où $\left.\nabla^{\prime} u^{*}\right|_{\Sigma} \in L^{q}(\Sigma)$ et en vertu d'un résultat classique (cf, [8]), on a $u_{\left.\right|_{\Sigma}} \in W^{1, q}(\Sigma)$.

- $\alpha>1$ :

D'après l'estimation (3.8), la suite $\nabla^{\prime} m^{\varepsilon} u^{\varepsilon}$ possède une sous suite notée encore $\nabla^{\prime} m^{\varepsilon} u^{\varepsilon}$ converge vers 0 dans $L^{q}(\Sigma)^{2}$, donc dans $L^{r}(\Sigma)^{2}$, et $\left.m^{\varepsilon} u^{\varepsilon} \rightarrow u^{*}\right|_{\Sigma}$ dans $W^{1, r}(\Sigma)$, alors $\left.\nabla^{\prime} u^{*}\right|_{\Sigma}=0$, d'où $\left.u^{*}\right|_{\Sigma}=C$.

D'où la preuve de la proposition 3.2.

Pour étudier le comportement asymptotique du problème $\left(\Pi^{\varepsilon}\right)$, on utilisera la méthode d'épiconvergence, (voir Annexe, definition A.1). 


\section{Comportement asymptotique}

Posons :

$$
\begin{aligned}
F^{\varepsilon}(u) & = \begin{cases}\frac{1}{p} \int_{\Omega_{\varepsilon}}|\nabla u|^{p}+\frac{1}{q \varepsilon^{\alpha}} \int_{B_{\varepsilon}}|\nabla u|^{q} & \text { si } u \in V^{\varepsilon} \\
+\infty & \text { si } u \in W_{0}^{1, r}(\Omega) \backslash V^{\varepsilon}\end{cases} \\
G(u) & =-\int_{\Omega} f u, \forall u \in W_{0}^{1, r}(\Omega)
\end{aligned}
$$

On note par $\tau_{f}$ la topologie faible sur $W_{0}^{1, r}(\Omega)$.

Théorème 4.1. Selon les valeurs de $\alpha$, il existe une fonctionnelle $F^{\alpha}: W_{0}^{1, r}(\Omega) \longrightarrow \mathbb{R} \cup\{+\infty\}$ telle que:

$$
\tau_{f}-\lim _{e} F^{\varepsilon}=F^{\alpha} \text { dans } W_{0}^{1, r}(\Omega)
$$

Où $F^{\alpha}$ est donnée par:

(1) Si $0 \leq \alpha<1$ :

$$
F^{\alpha}(u)= \begin{cases}\frac{1}{p} \int_{\Omega}|\nabla u|^{p} & \text { si } u \in W_{0}^{1, p}(\Omega) \\ +\infty & \text { si } u \in W_{0}^{1, r}(\Omega) \backslash W_{0}^{1, p}(\Omega)\end{cases}
$$

(2) Si $\alpha \geq 1$ :

$$
F^{\alpha}(u)= \begin{cases}\frac{1}{p} \int_{\Omega}|\nabla u|^{p}+\frac{2 m(\varphi) \eta(\alpha)}{q} \int_{\Sigma}\left|\nabla^{\prime} u_{\left.\right|_{\Sigma}}\right|^{q} & \text { si } u \in \mathbb{G}^{\alpha} \\ +\infty & \text { si } u \in W_{0}^{1, r}(\Omega) \backslash \mathbb{G}^{\alpha}\end{cases}
$$

Proof. $\quad$ a) On va déterminer l'épilimite supérieure :

Soit $u \in \mathbb{G}^{\alpha} \subset W_{0}^{1, r}(\Omega)$, comme $\mathbb{D}^{\alpha}$ est dense dans $\mathbb{G}^{\alpha}$, alors il existe une suite $\left(u^{n}\right)$ dans $\mathbb{D}^{\alpha}$ telle que $u^{n} \rightarrow u$ dans $\mathbb{G}^{\alpha}$ quand $n \rightarrow+\infty$, donc $u^{n} \rightarrow u$ dans $W_{0}^{1, r}(\Omega)$. Soit $\theta$ une fonction régulière vérifiant:

$$
\theta\left(x_{3}\right)=1 \text { si }\left|x_{3}\right| \leq 1, \theta\left(x_{3}\right)=0 \text { si }\left|x_{3}\right| \geq 2\left|\theta^{\prime}\left(x_{3}\right)\right| \leq 2 \forall x_{3} \in \mathbb{R},
$$

et

$$
\theta_{\varepsilon}(x)=\theta\left(\frac{x_{3}}{\varepsilon \varphi_{\varepsilon}}\right)
$$

On définit

$$
u^{\varepsilon, n}=\theta_{\varepsilon}(x) u_{\left.\right|_{\Sigma}}+\left(1-\theta_{\varepsilon}(x)\right) u^{n} .
$$

On a $u^{\varepsilon, n} \in V^{\varepsilon}$ et on montre aisément que $u^{\varepsilon, n} \rightarrow u^{n}$ dans $\mathbb{G}^{\alpha}$ quand $\varepsilon \longrightarrow 0$. Comme:

$$
F^{\varepsilon}\left(u^{\varepsilon, n}\right)=\frac{1}{p} \int_{\Omega_{\varepsilon}}\left|\nabla u^{\varepsilon, n}\right|^{p}+\frac{1}{q \varepsilon^{\alpha}} \int_{B_{\varepsilon}}\left|\nabla u^{\varepsilon, n}\right|^{q}
$$


Donc, on a :

$$
\begin{aligned}
& F^{\varepsilon}\left(u^{\varepsilon, n}\right)=\frac{1}{p} \int_{\left|x_{3}\right|>2 \varepsilon \varphi_{\varepsilon}}\left|\nabla u^{n}\right|^{p}+\frac{1}{p} \int_{\varepsilon \varphi_{\varepsilon}<\left|x_{3}\right|<2 \varepsilon \varphi_{\varepsilon}}\left|\nabla u^{\varepsilon, n}\right|^{p}+\frac{1}{q \varepsilon^{\alpha}} \int_{B_{\varepsilon}}\left|\nabla u^{\varepsilon, n}\right|^{q} \\
& =\frac{1}{p} \int_{\left|x_{3}\right|>2 \varepsilon \varphi_{\varepsilon}}\left|\nabla u^{n}\right|^{p}+\frac{1}{p} \int_{\varepsilon \varphi_{\varepsilon}<\left|x_{3}\right|<2 \varepsilon \varphi_{\varepsilon}}\left|\nabla u^{\varepsilon, n}\right|^{p}+\left.\frac{2 \varepsilon^{1-\alpha}}{q} \int_{\Sigma} \varphi_{\varepsilon}\left|\nabla^{\prime} u^{n}\right|_{\Sigma}\right|^{q} \text {. }
\end{aligned}
$$

Comme $\varphi_{\varepsilon}$ est bornée, alors on vérifie facilement que :

$$
\lim _{\varepsilon \longrightarrow 0}\left\{\int_{\varepsilon \varphi_{\varepsilon}<\left|x_{3}\right|<2 \varepsilon \varphi_{\varepsilon}} \underset{\left|\nabla u^{\varepsilon, n}\right|^{p}}{\mid}\right\}=0
$$

(1) Si $0 \leq \alpha \leq 1$ : Comme $\varphi_{\varepsilon} \rightarrow^{*} m(\varphi)$ dans $L^{\infty}(\Sigma), \varepsilon^{1-\alpha} \rightarrow \eta(\alpha)$, alors :

$$
\lim _{\varepsilon \rightarrow 0} \frac{2 \varepsilon^{1-\alpha}}{q} \int_{\Sigma} \varphi_{\varepsilon}\left|\nabla u_{\mid \Sigma}^{n}\right|^{q}=\frac{2 m(\varphi) \eta(\alpha)}{q} \int_{\Sigma}\left|\nabla^{\prime} u_{\mid \Sigma}^{n}\right|^{q} .
$$

Donc par passage à la limite supérieure on a

$$
\begin{aligned}
\limsup _{\varepsilon \rightarrow 0} F^{\varepsilon}\left(u^{\varepsilon, n}\right) & =\limsup _{\varepsilon \rightarrow 0}\left(\frac{1}{p} \int_{\left|x_{3}\right|>2 \varepsilon \varphi_{\varepsilon}}\left|\nabla u^{n}\right|^{p}+\left.\frac{2 \varepsilon^{1-\alpha}}{q} \int_{\Sigma} \varphi_{\varepsilon}\left|\nabla^{\prime} u^{n}\right|_{\Sigma}\right|^{q}\right) \\
& =\frac{1}{p} \int_{\Omega}\left|\nabla u^{n}\right|^{p}+\left.\frac{2 m(\varphi) \eta(\alpha)}{q} \int_{\Sigma}\left|\nabla^{\prime} u^{n}\right|_{\Sigma}\right|^{q} .
\end{aligned}
$$

(2) $\alpha>1$ : Donc par passage à la limite supérieure on a

$$
\begin{aligned}
\limsup _{\varepsilon \rightarrow 0} F^{\varepsilon}\left(u^{\varepsilon, n}\right) & =\limsup _{\varepsilon \rightarrow 0}\left(\frac{1}{p} \int_{\left|x_{3}\right|>2 \varepsilon \varphi_{\varepsilon}}\left|\nabla u^{n}\right|^{p}\right) \\
& =\frac{1}{p} \int_{\Omega}\left|\nabla u^{n}\right|^{p} .
\end{aligned}
$$

Comme $u^{n} \rightarrow u$ dans $\mathbb{G}^{\alpha}$ quand $n \longrightarrow+\infty$, donc en vertu d'un résultat classique, lemme de diagonalisation, (cf, [2, Lemma 1.15 p. 32]), il existe une application $n(\varepsilon): \mathbb{R}^{+} \longrightarrow \mathbb{N}$ croissante vers $+\infty$ quand $\varepsilon \rightarrow 0$ telle que $u^{\varepsilon, n(\varepsilon)} \rightarrow u$ dans $\mathbb{G}^{\alpha}$ quand $\varepsilon \rightarrow 0$.

En faisant tendre $n$ vers $+\infty$, on aura :

(1) Pour $0 \leq \alpha \leq 1$ :

$$
\begin{aligned}
\limsup _{\varepsilon \rightarrow 0} F^{\varepsilon}\left(u^{\varepsilon, n(\varepsilon)}\right) & \leq \limsup _{n \rightarrow+\infty} \limsup _{\varepsilon \rightarrow 0} F^{\varepsilon}\left(u^{\varepsilon, n}\right) \\
& \leq \frac{1}{p} \int_{\Omega}|\nabla u|^{p}+\frac{2 m(\varphi) \eta(\alpha)}{q} \int_{\Sigma}\left|\nabla^{\prime} u_{\left.\right|_{\Sigma}}\right|^{q} .
\end{aligned}
$$

(2) Pour $\alpha>1$ :

$$
\begin{aligned}
\limsup _{\varepsilon \rightarrow 0} F^{\varepsilon}\left(u^{\varepsilon, n(\varepsilon)}\right) & \leq \limsup _{n \rightarrow+\infty} \limsup _{\varepsilon \rightarrow 0} F^{\varepsilon}\left(u^{\varepsilon, n}\right) \\
& \leq \frac{1}{p} \int_{\Omega}|\nabla u|^{p}
\end{aligned}
$$


Si $u \in W_{0}^{1, r}(\Omega) \backslash \mathbb{G}^{\alpha}$, alors il est clair que pour tout $u^{\varepsilon} \in V^{\varepsilon}, u^{\varepsilon} \rightarrow u$, on a

$$
\limsup _{\varepsilon \rightarrow 0} F^{\varepsilon}\left(u^{\varepsilon}\right) \leq+\infty
$$

b) On va déterminer l'épilimite inférieure:

Soient $u \in \mathbb{G}^{\alpha}$ et une suite $\left(u^{\varepsilon}\right)$ dans $V^{\varepsilon}$ tels que $u^{\varepsilon} \rightarrow u$ dans $W_{0}^{1, r}(\Omega)$, donc

$$
\chi_{\Omega_{\varepsilon}} \nabla u^{\varepsilon} \rightarrow \nabla u \text { dans } L^{r}(\Omega)^{3} .
$$

(1) Si $\alpha \neq 1$ :

Si $\liminf _{\varepsilon \rightarrow 0} F^{\varepsilon}\left(u^{\varepsilon}\right)=+\infty$, rien à démontrer, car

$$
\frac{1}{p} \int_{\Omega}|\nabla u|^{p} \leq+\infty
$$

Sinon, $\liminf _{\varepsilon \rightarrow 0} F^{\varepsilon}\left(u^{\varepsilon}\right)<+\infty$, il existe une sous suite de $F^{\varepsilon}\left(u^{\varepsilon}\right)$ notée encore $F^{\varepsilon}\left(u^{\varepsilon}\right)$ et une constante $C>0$ telle que $F^{\varepsilon}\left(u^{\varepsilon}\right) \leq C$, ce qui entrane que

$$
\frac{1}{p} \int_{\Omega_{\varepsilon}}\left|\nabla u^{\varepsilon}\right|^{p} \leq C
$$

donc $\chi_{\Omega_{\varepsilon}} \nabla u^{\varepsilon}$ est bornée dans $L^{p}(\Omega)^{3}$, pour une sous suite de $\chi_{\Omega_{\varepsilon}} \nabla u^{\varepsilon}$, notée encore $\chi_{\Omega_{\varepsilon}} \nabla u^{\varepsilon}$, il existe un élément $w^{0} \in L^{p}(\Omega)^{3}$ tel que

$$
\chi_{\Omega_{\varepsilon}} \nabla u^{\varepsilon} \rightarrow w^{0} \text { dans } L^{p}(\Omega)^{3},
$$

en vertu de (4.4), on aura $w^{0}=\nabla u$.

Comme

$$
F^{\varepsilon}\left(u^{\varepsilon}\right) \geq \frac{1}{p} \int_{\Omega_{\varepsilon}}\left|\nabla u^{\varepsilon}\right|^{p} .
$$

Par passage à la limite inférieure, on obtient

$$
\liminf _{\varepsilon \rightarrow 0} F^{\varepsilon}\left(u^{\varepsilon}\right) \geq \frac{1}{p} \int_{\Omega}|\nabla u|^{p} .
$$

(2) Si $\alpha=1$ :

Si $\liminf _{\varepsilon \rightarrow 0} F^{\varepsilon}\left(u^{\varepsilon}\right)=+\infty$, rien à démontrer, car

$$
\frac{1}{p} \int_{\Omega}|\nabla u|^{p}+\frac{2 m(\varphi) \eta(\alpha)}{q} \int_{\Sigma}\left|\nabla^{\prime} u_{\left.\right|_{\Sigma}}\right|^{q} \leq+\infty .
$$

Sinon, $\liminf _{\varepsilon \rightarrow 0} F^{\varepsilon}\left(u^{\varepsilon}\right)<+\infty$, il existe une sous suite de $F^{\varepsilon}\left(u^{\varepsilon}\right)$ notée encore $F^{\varepsilon}\left(u^{\varepsilon}\right)$ et une constante $C>0$, telle que $F^{\varepsilon}\left(u^{\varepsilon}\right) \leq C$, ce qui entrane que

$$
\begin{gathered}
\frac{1}{p} \int_{\Omega_{\varepsilon}}\left|\nabla u^{\varepsilon}\right|^{p} \leq C, \\
\frac{1}{q \varepsilon^{\alpha}} \int_{B_{\varepsilon}}\left|\nabla u^{\varepsilon}\right|^{q} \leq C .
\end{gathered}
$$


donc $u^{\varepsilon}$ vérifie les hypothèses du lemme 3.3, et en vertu de ce dernier, $\nabla^{\prime} m^{\varepsilon} u^{\varepsilon}$ est bornée dans $L^{q}(\Sigma)^{2}$, donc il existe un élément $u_{1} \in L^{q}(\Sigma)^{2}$, et une sous suite de $\nabla^{\prime} m^{\varepsilon} u^{\varepsilon}$, notée encore $\nabla^{\prime} m^{\varepsilon} u^{\varepsilon}$, tel que $\nabla^{\prime} m^{\varepsilon} u^{\varepsilon} \rightarrow u_{1}$ dans $L^{q}(\Sigma)^{2}$ donc dans $L^{r}(\Sigma)^{2}$, et comme $u_{\left.\right|_{\Sigma}}^{\varepsilon} \rightarrow u_{\left.\right|_{\Sigma}}$ dans $L^{r}(\Sigma)$, d'après (3.4) et (4.5), on a $m^{\varepsilon} u^{\varepsilon} \rightarrow u_{\left.\right|_{\Sigma}}$ dans $L^{r}(\Sigma)$, donc $m^{\varepsilon} u^{\varepsilon} \rightarrow u_{\left.\right|_{\Sigma}}$ dans $W^{1, r}(\Sigma)$, et par suite $u_{1}=\nabla^{\prime} u_{\left.\right|_{\Sigma}}$, donc $\nabla^{\prime} m^{\varepsilon} u^{\varepsilon} \rightarrow \nabla^{\prime} u_{\left.\right|_{\Sigma}}$ dans $L^{q}(\Sigma)^{2}$.

Comme $\chi_{\Omega_{\varepsilon}} \nabla u^{\varepsilon}$ est bornée dans $L^{p}(\Omega)^{3}$, pour une sous suite de $\chi_{\Omega_{\varepsilon}} \nabla u^{\varepsilon}$, notée encore $\chi_{\Omega_{\varepsilon}} \nabla u^{\varepsilon}$, il existe un élément $w^{0} \in L^{p}(\Omega)^{3}$, tel que :

$$
\chi_{\Omega_{\varepsilon}} \nabla u^{\varepsilon} \rightarrow w^{0} \text { dans } L^{p}(\Omega)^{3},
$$

en vertu de (4.4), on aura $w^{0}=\nabla u$.

On a

$$
\begin{aligned}
F^{\varepsilon}\left(u^{\varepsilon}\right) & \geq \frac{1}{p} \int_{\Omega_{\varepsilon}}\left|\nabla u^{\varepsilon}\right|^{p}+\frac{1}{q \varepsilon^{\alpha}} \int_{B_{\varepsilon}}\left|\nabla u^{\varepsilon}\right|^{q} \\
& \geq \frac{1}{p} \int_{\Omega_{\varepsilon}}\left|\nabla u^{\varepsilon}\right|^{p}+\frac{2 \varepsilon^{1-\alpha}}{q} \int_{\Sigma} \varphi_{\varepsilon}\left|\nabla^{\prime} m^{\varepsilon} u^{\varepsilon}\right|^{q} .
\end{aligned}
$$

D'après l'inégalité de sous différentiabilité de $v \longrightarrow \frac{2 \varepsilon^{1-\alpha}}{q} \int_{\Sigma} \varphi_{\varepsilon}|v|^{q}, \forall v \in L^{q}(\Sigma)^{2}$, on a

$$
F^{\varepsilon}\left(u^{\varepsilon}\right) \geq \frac{1}{p} \int_{\Omega_{\varepsilon}}\left|\nabla u^{\varepsilon}\right|^{p}+\frac{2 \varepsilon^{1-\alpha}}{q} \int_{\Sigma} \varphi_{\varepsilon}\left|\nabla^{\prime} u_{\left.\right|_{\Sigma}}\right|^{q}+\frac{2 \varepsilon^{1-\alpha}}{q} \int_{\Sigma} \varphi_{\varepsilon}\left|\nabla^{\prime} u_{\left.\right|_{\Sigma}}\right|^{q-2} \nabla^{\prime} u_{\left.\right|_{\Sigma}}\left(\nabla^{\prime} m^{\varepsilon} u^{\varepsilon}-\nabla^{\prime} u_{\left.\right|_{\Sigma}}\right) .
$$

En vertu du lemme A.1, on a $\varphi_{\varepsilon} \rightarrow m(\varphi)$ dans $L^{q^{\prime}}(\Sigma)$, donc par passage à la limite inférieure, on obtient

$$
\liminf _{\varepsilon \rightarrow 0} F^{\varepsilon}\left(u^{\varepsilon}\right) \geq \frac{1}{p} \int_{\Omega}|\nabla u|^{p}+\frac{2 m(\varphi) \eta(\alpha)}{q} \int_{\Sigma}\left|\nabla^{\prime} u_{\left.\right|_{\Sigma}}\right|^{q} .
$$

Si $u \in W_{0}^{1, r} \backslash \mathbb{G}^{\alpha}$, et $u^{\varepsilon} \in V^{\varepsilon}$, telle que $u^{\varepsilon} \rightarrow u$ dans $W_{0}^{1, r}(\Omega)$.

Supposons que

$$
\liminf _{\varepsilon \rightarrow 0} F^{\varepsilon}\left(u^{\varepsilon}\right)<+\infty .
$$

Donc il existe une constante $C>0$ et une sous suite de $F^{\varepsilon}\left(u^{\varepsilon}\right)$, notée encore $F^{\varepsilon}\left(u^{\varepsilon}\right)$, telle que

$$
F^{\varepsilon}\left(u^{\varepsilon}\right)<C .
$$

En faisant la même démarche comme celle du cas $u \in \mathbb{G}^{\alpha}$, on obtient que $u \in \mathbb{G}^{\alpha}$ ce qui contredit le fait que $u \notin \mathbb{G}^{\alpha}$, donc on a

$$
\liminf _{\varepsilon \rightarrow 0} F^{\varepsilon}\left(u^{\varepsilon}\right)=+\infty .
$$

D'où la preuve du théorème 4.1 .

Dans la suite on s'interesse à la détermination du problème limite associé au problème $\left(\Pi^{\varepsilon}\right)$, lorsque en faisant tendre $\varepsilon$ vers zero. D'après les résultats sur l'épiconvergence (voir Annexe, théorème A.3 et proposition A.2) et le théorème 4.1, et en vertu de la $\tau_{f}$-continuité de $G$ dans $W_{0}^{1, r}(\Omega)$, on a $F^{\varepsilon}+G \tau_{f}$-épiconverge vers $F^{\alpha}+G$ dans $W_{0}^{1, r}(\Omega)$. 
Proposition 4.2. Pour tout $f \in L^{r^{\prime}}(\Omega)$ et selon les valeurs du paramètre $\alpha$, il existe $u^{*} \in W_{0}^{1, r}(\Omega)$ satisfaisant $\grave{a}$

$$
\begin{array}{r}
u^{\varepsilon} \rightarrow u^{*} \text { dans } W_{0}^{1, r}(\Omega), \\
F^{\alpha}\left(u^{*}\right)+G\left(u^{*}\right)=\inf _{v \in \mathbb{G}^{\alpha}}\left\{F^{\alpha}(v)+G(v)\right\} .
\end{array}
$$

Proof. En vertu du lemme 3.1, la famille $u^{\varepsilon}$ est bornée dans $W_{0}^{1, r}(\Omega)$, et par suite elle possède une $\tau_{f}$-valeur d'adhérence $u^{*}$ dans $W_{0}^{1, r}(\Omega)$. Et d'après un résultat classique d'épilimite (voir Annexe, théorème A.3), on a $u^{*}$ est une solution du problème

$$
\left(\Pi^{\alpha}\right) \quad \inf _{v \in W_{0}^{1, r}(\Omega)}\left\{F^{\alpha}(v)+G(v)\right\} .
$$

Comme $F^{\alpha}$ vaut $+\infty$ sur $W_{0}^{1, r}(\Omega) \backslash \mathbb{G}^{\alpha}$, alors (4.6) devient

$$
\left(\Pi^{\alpha}\right) \quad \inf _{v \in \mathbb{G}^{\alpha}}\left\{F^{\alpha}(v)+G(v)\right\} .
$$

En vertu de l'unicité de solutions du problème $\left(\Pi^{\alpha}\right)$, donc $u^{\varepsilon}$ admet une unique $\tau_{f}$-valeur d'adhérence $u^{*}$, et par conséquent $u^{\varepsilon} \rightarrow u^{*}$ dans $W_{0}^{1, r}(\Omega)$.

Commentaire 4.1. On montre que le comportement asymptotique d'une structure constituée de deux milieux de conductivité constante réunis par une bande oscillante d'épaisseur $\varepsilon$, non linéaire dont la conductivité dépend des puissances négatives de $\varepsilon$, est décrit par un problème avec interface $\Sigma,(\Sigma$ l'interface moyenne de la bande). Suivant les puissances de $\varepsilon$, à l'interface $\Sigma$, on a la chaleur est continue, un problème bidimensionnel ou la chaleur est constante.

\section{Traitement numÉRiQue}

Nous avons montré que pour $\varepsilon$ assez petit, la solution $u^{\varepsilon}$ du problème $\left(\Pi^{\varepsilon}\right)$, dans un certain sens, approche la solution $u^{*}$ du problème limite $\left(\Pi^{\alpha}\right)$. Dans cette section nous intéressons au traitement numérique des problèmes $\left(\Pi^{\varepsilon}\right)$ et $\left(\Pi^{\alpha}\right)$, afin d'illustrer les résultats obtenus dans l'étude théorique.

Prenons les problèmes $\left(\Pi^{\varepsilon}\right)$ et $\left(\Pi^{\alpha}\right)$, avec :

$$
\begin{aligned}
& \Omega=]-1,1[\times]-1,1[, \\
& f(x, y)=0.01 \exp \left(-x^{2}-y^{2}\right), \\
& \varphi_{\varepsilon}(x)=1.2+\sin \left(\pi \frac{x}{\varepsilon}\right) .
\end{aligned}
$$

On résout numériquement les problèmes $\left(\Pi^{\varepsilon}\right)$ et $\left(\Pi^{\eta}\right)$, par le langage scientifique FreeFem++ (voir [6]), par la méthode des éléments finis et en utilisant la méthode de Newton, avec $p=3.5, q=2.5$ et $\varepsilon=1 e-06$, et on aura les résultats suivant : 



Figure 5.1. La solution du problème $\left(\Pi^{\varepsilon}\right)$ ( à gauche ), La solution du problème limite (à droite), pour $\alpha=0.01$.
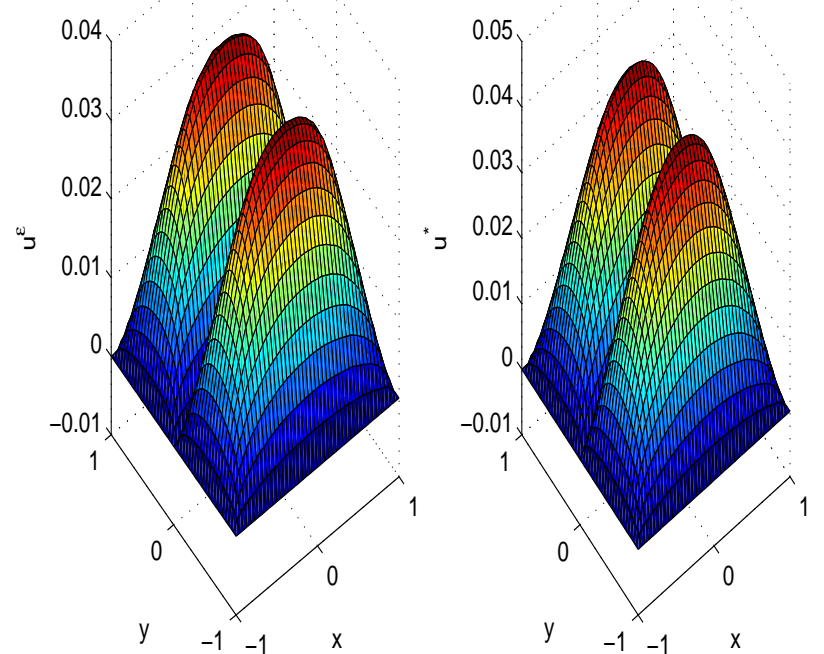

Figure 5.2. La solution du problème $\left(\Pi^{\varepsilon}\right)$ ( à gauche ), La solution du problème limite (à droite), pour $\alpha=1$. 

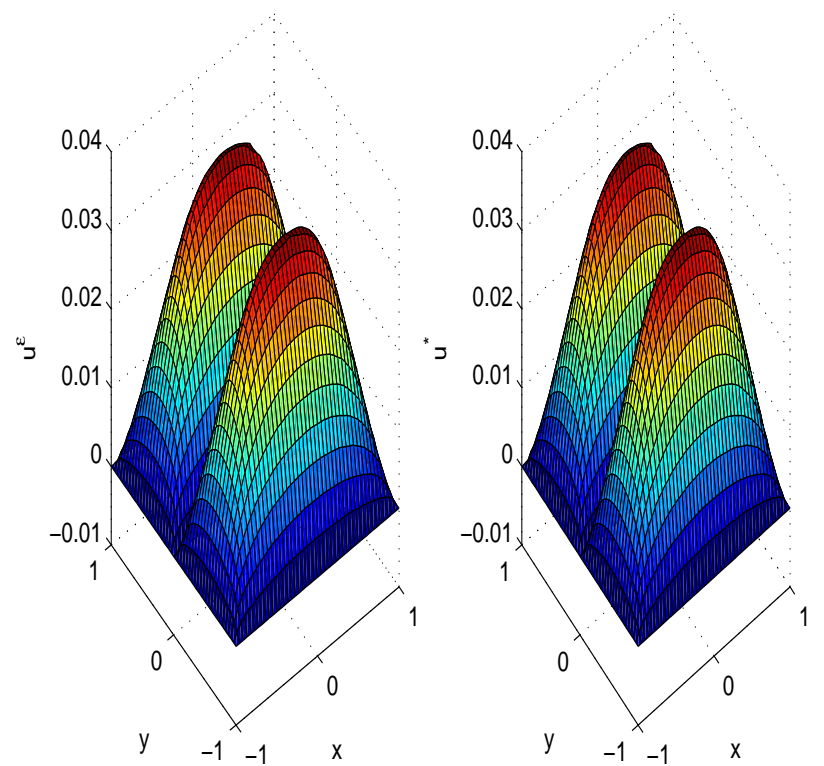

Figure 5.3. La solution du problème $\left(\Pi^{\varepsilon}\right)$ ( à gauche ), La solution du problème limite (à droite), pour $\alpha=4.5$.

La figure 5.1 (resp. 5.2, 5.3) montre que la solution du problème $\left(\Pi^{\varepsilon}\right)$ approche celle du problème limite $\left(\Pi^{\alpha}\right),(\alpha=0.01,1$ ou 4.5$)$ avec une erreur d'ordre $7 e-05$ (resp. $\left.1 e-04,5 e-10\right)$.

\section{A. AnNeXe}

Definition A.1. ( [2], Definition 1.9) Soit $(X, \tau)$ un espace métrique, $\left(F^{\varepsilon}\right)_{\varepsilon}$ et $F$ sont des fonctionnelles de $X$ dans $\mathbb{R} \cup\{+\infty\}$. On dit que $F^{\varepsilon}$ épiconvergence vers $F$ dans $(X, \tau)$, noté $\tau-\lim _{e} F^{\varepsilon}=F$, si

- $\forall x \in X, \exists x_{\varepsilon}^{0}, x_{\varepsilon}^{0} \stackrel{\tau}{\rightarrow} x, \limsup _{\varepsilon \rightarrow 0} F^{\varepsilon}\left(x_{\varepsilon}^{0}\right) \leq F(x)$,

- $\forall x \in X, \forall x_{\varepsilon}, x_{\varepsilon} \stackrel{\tau}{\rightarrow} x, \liminf _{\varepsilon \rightarrow 0} F^{\varepsilon}\left(x_{\varepsilon}\right) \geq F(x)$.

Proposition A.2. ( [2], p.40) Soient $F^{\varepsilon}, F, G: X \longrightarrow \mathbb{R} \cup\{+\infty\}$, telles que $\tau-\lim _{e} F^{\varepsilon}=F$ et $G$ est $\tau-$ continue, alors

$$
\tau-\lim _{e}\left(F^{\varepsilon}+G\right)=F+G .
$$

Cette méthode d'épiconvergence est un cas special de la méthode $\Gamma$-convergence introduite par De Giorgi (1979). C'est bien convenu à l'analyse asymptotique de suites de problèmes de minimisation depuis qu'on a le résultat fondamental suivant.

Théorème A.3. ( [2], théorème 1.10) Soient $F^{\varepsilon}, F: X \longrightarrow \mathbb{R} \cup\{+\infty\}$, supposons que

(1) Il existe une suite $\left(\bar{u}^{\varepsilon}\right) \subset X$ telle que

$$
\inf _{u \in X} F^{\varepsilon}(u)=F^{\varepsilon}\left(\bar{u}^{\varepsilon}\right) .
$$

(2) La suite $\left(\bar{u}^{\varepsilon}\right)$ est $\tau$-relativement compacte.

(3) $\tau-\lim _{e} F^{\varepsilon}=F$. 
alors toute $\tau$-valeur d'adhérence $\bar{u}$ de $\left(\bar{u}^{\varepsilon}\right)$ satisfait $\grave{a}$

$$
F(\bar{u})=\inf _{u \in X} F(u)
$$

Lemme A.1. Soit $\varphi \in L^{\infty}(\Sigma), Y$-périodique, $\left.Y=\right] 0,1[\times] 0,1[$, posons

$$
\varphi_{\varepsilon}(x)=\varphi\left(\frac{x}{\varepsilon}\right), \text { pour } \varepsilon>0 \text { assez petit, }
$$

alors

$$
\left\{\begin{array}{lc}
\varphi_{\varepsilon} \rightarrow m(\varphi) & \text { dans } L^{s}(\Sigma) \text { pour } 1 \leq s<\infty \\
\varphi_{\varepsilon} \rightarrow^{*} m(\varphi) & \text { dans } L^{\infty}(\Sigma) .
\end{array}\right.
$$

Proof. Comme $\varphi_{\varepsilon}$ est $\varepsilon Y$-périodique, alors on a

$$
\left\{\begin{array}{cc}
\varphi_{\varepsilon} \rightarrow m(\varphi) & \text { dans } L^{s}(\Sigma) \text { pour } 1 \leq s<\infty \\
\varphi_{\varepsilon} \rightarrow^{*} m(\varphi) & \text { dans } L^{\infty}(\Sigma)
\end{array}\right.
$$

Or $\varphi$ est bornée $p . p$. dans $\Sigma$, alors pour tout $s \geq 1$, il existe une constante $C>0$, telle que

$$
\begin{aligned}
\int_{\Sigma}\left|\varphi_{\varepsilon}-m(\varphi)\right|^{s} & \leq C \int_{\Sigma}\left|\varphi_{\varepsilon}-m(\varphi)\right| \\
& \leq C\left(\int_{\varphi \geq m(\varphi)}\left(\varphi_{\varepsilon}-m(\varphi)\right)-\int_{\varphi \leq m(\varphi)}\left(\varphi_{\varepsilon}-m(\varphi)\right)\right)
\end{aligned}
$$

Donc par passage à la limite dans (A.2), on aura

$$
\varphi_{\varepsilon} \rightarrow m(\varphi) \text { dans } L^{s}(\Sigma) \text { pour } 1 \leq s<\infty
$$

D'où la preuve du lemme A.1.

\section{REFERENCES}

[1] Ait Moussa A. Eт Licht C., Comportement asymptotique d'une plaque mince non linéaire, J. Math du Maroc, No2, 1994, p.1-16.

[2] Аттоuch H., Variational Convergence for Functions and Operators. Pitman Advance Publishing Program, (1984).

[3] Brezis H., Analyse fonctionnelle, Théorie et Applications, Masson(1992).

[4] BRillard A.,Asymptotic analysis of nonlinear thin layers, International Series of Numerical Mathematics, Vol $123,1997$.

[5] Ganghoffer J. F., Brillard A. And Schultz J., Modelling of mechanical behaviour of joints bonded by a nonlinear incompressible elastic adhesive, European Journal of Mechanics. A/Solids. Vol 16, No2, 255-276. 1997.

[6] Hecht F., Pironneau O., Le Hyaric A., Ohtsuka K., FreeFem++ Manual, downloadable at http://www.Freefem.org.

[7] Phum Huy H. and Sanchez-Palencia E.,Phénoménes de transmission à travers des couches minces de conductivité élevée, Journal of Mathematical Analysis and Applications, 47, pp.284-309,1974.

[8] Tемам R.-Problèmes mathématiques en plasticité, Ed. Gauthier Villars, Paris, 1983. 